\title{
NONPRODUCT TYPE ANALYTIC TUHF ALGEBRAS
}

\author{
BELISARIO A. VENTURA
}

\begin{abstract}
We construct examples of nonproduct type real valued cocycles on a UHF groupoid, and show that the analytic triangular algebras associated to those cocycles, can only correspond to nonproduct type cocycles.
\end{abstract}

\section{INTRODUCTION}

Suppose that $\mathscr{A}$ is a UHF algebra. The usual approach to UHF algebras considers $\mathscr{A}$ as a union of a nested sequence of matrix algebras. However, $\mathscr{A}$ can also be realized as the $\mathrm{C}^{*}$-algebra of a groupoid $R$, which is actually an equivalence relation on the Cantor set (cf. [R], page 128). Regarding UHF algebras as groupoid $\mathrm{C}^{*}$-algebras is useful for the study of analytic triangular subalgebras of $\mathscr{A}$ (or analytic TUHF algebras), and automorphisms of $\mathscr{A}$ associated with real-valued continuous cocycles on $R$ (cf. [B], [PPW 2], [V]). Given a cocycle $d$ on the groupoid $R$ (that is a homomorphism $d: R \rightarrow \mathbb{R}$, where $\mathbb{R}$ is the set of real numbers) that vanishes only on the unit space of $R$, there is an analytic TUHF algebra of $\mathscr{A}$ associated with $d$, and there is also a one-parameter group of automorphisms induced by $d$. The TUHF algebra associated with $d$ is the algebra supported on the subset of $R$ where the cocycle is non-negative (cf. [V], Section 5, [PPW2], Section 1). The oneparameter automorphism group induced by $d$, is given, for each $t \in \mathbb{R}$, by pointwise multiplication by $\exp (i t d(\cdot, \cdot))$ ([R], Proposition II.5.1).

As far as these associated objects is concerned, the simplest class of cocycles is given by those of product type (unexplained terminology will be defined in the next section). They correspond to a factorization of $\mathscr{A}$ as an infinite tensor product of matrix algebras, and this factorization provides sufficient eigenvalues and eigenvectors for the automorphism group, as well as canonical finitedimensional approximants for the TUHF algebra.

In this paper, we continue the study of analytic TUHF algebras begun in [V]. Our emphasis here is on cocycles that are not of product type. In fact, we give some examples of such cocycles. These examples are all coboundaries, so their associated one-parameter groups of automorphisms are inner. Having examples

Received by the editors January 12, 1994 and, in revised form, June 13, 1994.

1991 Mathematics Subject Classification. Primary 46L40, 46L55, 22A22; Secondary 46H20, 46L05, $20 \mathrm{~L} 29$.

Key words and phrases. Nonproduct type cocycle, analytic triangular UHF algebras, UHF groupoid, nonproduct type automorphism.

Partially supported by a Faculty Professional Development Grant (Summer Fellowship 1992) from California State University, San Bernardino. 
of both kinds of cocycles, prompts the following question: Can an analytic TUHF algebra correspond only to cocycles of product type (resp. nonproduct type)? We show that the answer to this question is affirmative in the case of nonproduct type cocycles. In fact, the analytic TUHF algebras induced by our examples of nonproduct type cocycles can only be associated with cocycles of that kind.

After we found the examples of nonproduct type cocycles described in this paper, B. Solel showed us an example [S] he had found of a cocycle that is not of product type and that corresponds to the standard embedding TUHF algebra ([V], Example 6.1). The standard embedding TUHF algebra corresponds canonically to a product type cocycle ([PPW2], Theorem 2.2), thus Solel's example shows that it is also possible for an analytic TUHF algebra to be associated to both kinds of cocycles.

Regarding the automorphisms implemented by the nonproduct type cocycles described above, our examples implement nonproduct type actions of $\mathbb{R}$ on UHF algebras, while Solel's example implements a nonproduct type action of $\mathbb{Z}$. As far as we know, these constitute the first examples of nonproduct type actions of $\mathbb{R}$ and $\mathbb{Z}$. Previously, Kishimoto $[\mathrm{K}]$ and Handelman and Rossmann [HR] have constructed nonproduct type actions for finite groups and compact groups, respectively.

This paper is organized as follows. Section 2 contains the preliminaries and the notational conventions. In Section 3 we give the examples of nonproduct type cocycles to which we referred before. These examples are divided into two groups (Example 3.1 and Example 3.6). In both cases we establish that the cocycles are not of product type by showing that they are not locally constant (Theorem 3.2 and Theorem 3.7). We also indicate how to obtain the corresponding nonproduct type one-parameter automorphism groups. Sections 4 and 5 are devoted to the TUHF algebras associated with the cocycles given in Section 3. In Section 4 we construct these algebras as inductive limits of finite-dimensional upper triangular matrix algebras by exhibiting the embeddings ((4.1) and (4.6), respectively) and then proving, in Theorem 4.2 (resp. Theorem 4.7), that the embedding (4.1) (resp. (4.6)) induces the same TUHF algebra as the cocycle of Example 3.1 (resp. 3.6). In Section 5 we show that the TUHF algebras constructed in Section 4 can only be associated to nonproduct type cocycles. These results, Corollary 5.5 and Theorem 5.10, are obtained as consequences of Theorem 5.3, which is the main result of the section. In Section 5 we also introduce the term Nonproduct Type Analytic TUHF algebras, to denote those analytic TUHF algebras that can only correspond to nonproduct type cocycles. With this terminology, Theorem 5.3, gives sufficient conditions for membership in the class of Nonproduct Type Analytic TUHF's.

Although the emphasis of this paper is on UHF algebras and product type cocycles, the proof of Theorem 5.3 can be applied to AF algebras giving as a consequence that the strongly maximal triangular $\mathrm{AF}$ algebras that are not generated by their order preserving normalizers cannot correspond to a locally constant cocycle. In Remark 5.13 we point out this fact, and indicate the changes that are to be made in the statement of Theorem 5.3, in order to obtain an AF version of it. Locally constant cocycles on AF groupoids, although not considered in $\mathrm{AF} \mathrm{C}^{*}$-algebra theory, are gaining importance within triangular $\mathrm{AF}$ algebra theory, as recent work of A. Donsig and T. Hudson shows [DH]. 


\section{BASIC DEFINITIONS AND NOTATION}

Let $X=\prod_{i=1}^{\infty} Y_{i}$, where $Y_{i}$ is a finite set for every $i$, with

$$
\left|Y_{1}\right|=N(1), \quad\left|Y_{n+1}\right|=N(n+1) / N(n)
$$

(here $|Y|$ is the cardinality of the set $Y$ ).

We denote by $R$, the Glimm groupoid on $X$, namely for $x=\left(x_{n}\right)$ and $y=\left(y_{n}\right), x \sim y$ iff $x_{n}=y_{n}$ for all but finitely many $n^{\prime} s$. We identify $X$ with the unit space $X \times X$ of $R$. The topology of $R$ is given by considering the graphs $\hat{\sigma}$ of all the partial homeomorphisms $\sigma$ that change only finitely many coordinates of the points of $X$. The collection of such graphs forms a base of compact open sets for the topology of $R$ (cf. [R], II.4.15 and I.2.13, [V], Section 2).

$Z^{1}(R, \mathbb{R})$ will denote the set of continuous, real valued one-cocycles on $R$, that is, the continuous homomorphisms $d: R \rightarrow \mathbb{R}$. We will refer to all the elements of $Z^{1}(R, \mathbb{R})$ simply as cocycles. We assume that all the cocycles we consider vanish only on the unit space (or diagonal) $X$ of $R$, that is, $d$ satisfies $d^{-1}(0)=X$. We recall that a cocycle $d$ is a coboundary, if there is a continuous function $b: X \rightarrow \mathbb{R}$ such that $d(x, y)=b(y)-b(x)$. In this case we say that $d$ is implemented by $b$, and we will use the notation $d=\delta b$ to refer to this relationship.

Definition 2.1. $d \in Z^{1}(R, \mathbb{R})$ is of product type if it is of the form

$$
d(x, y)=\sum_{i=1}^{\infty} d_{i}\left(x_{i}, y_{i}\right), \quad \text { where } d_{i} \in Z^{1}\left(Y_{i} \times Y_{i}, \mathbb{R}\right) .
$$

Let $\mathscr{A}=C^{*}(R)$ be the UHF algebra of the Glimm groupoid $R$ ([R], Chapter II and [V], Section 2). That is $\mathscr{A}$ is the closure, under a suitable $\mathrm{C}^{*}$-norm, of the span of the characteristic functions of the graphs $\hat{\sigma}$ that form a base for the topology of $R$ mentioned above. If we view $\mathscr{A}$ as a union of an increasing collection of matrix algebras, each matrix unit $v$ in the finite dimensional approximants induces a partial homeomorphism $\sigma_{v}$ that changes only finitely many coordinates in some points of $X$, and the matrix unit $v$ can be regarded as the characteristic function $\chi(\hat{v})$ of the graph $\hat{v}$ of the partial homeomorphism $\sigma_{v}$. We will say that $\hat{v}$ is the support of $v$. Given a matrix unit $v$, the partial homeomorphism $\sigma_{v}$ induced by $v$ maps the support of the final projection of $v, \widehat{v v^{*}}$ (which coincides with the range of the $R$-set $\hat{v}$ ), to the support of the initial projection of $v, \widehat{v^{*} v}$ (which coincides with the source of the $R$-set $\hat{v}$ ). This set of correspondences establishes the connection between the two approaches to UHF algebras.

The main simplification introduced by a product type cocycle is summarized in the next lemma.

Lemma 2.2 (Lemma 5.4 of [V]). Let $d$ be a cocycle of product type on $R$. There is a system of matrix units $\left\{e_{i j}^{(n)}\right\}$ in $\mathscr{A}=C^{*}(R)$, such that $d$ is constant on $\hat{e}_{i j}^{(n)}$, the support of $e_{i j}^{(n)}$. It follows that $d$ is locally constant.

Outline of proof. Given $\vec{x}=\left(x_{1}, \ldots, x_{n}\right), \vec{y}=\left(y_{1}, \ldots, y_{n}\right) \in \prod_{i=1}^{n} Y_{i}$, we consider the partial homeomorphism $\sigma(\vec{x}, \vec{y})$ of $X$, defined on those $w=$ 
$\left(w_{i}\right)$ for which $w_{i}=x_{i}$ for $1 \leq i \leq n$, and given by $\sigma(\vec{x}, \vec{y})(w)=z$, where $w=\left(w_{i}\right), \quad z=\left(z_{i}\right), w_{i}=x_{i}$ for $1 \leq i \leq n$ and

$$
z_{i}= \begin{cases}y_{i} & \text { if } 1 \leq i \leq n \\ w_{i} & \text { if } i>n\end{cases}
$$

We can verify that on $\sigma(\widehat{\vec{x},} \vec{y})$ (the graph of $\sigma(\vec{x}, \vec{y})$ ), $d$ has the constant value $\sum_{i=1}^{n} d_{i}\left(x_{i}, y_{i}\right)$, and that

$$
\mathfrak{R}=\left\{\sigma(\widehat{\vec{x}, \vec{y}}): \vec{x}, \vec{y} \in \prod_{i=1}^{n} Y_{i}, n=1,2, \ldots\right\}
$$

supports a system of matrix units for $\mathscr{A}$.

Definition 2.3. The system of matrix units supported on the sets in $\mathfrak{R}$ is called the system of matrix units induced by the cocycle $d$.

Consequently, the set $\mathfrak{R}$ forms a base of compact open sets for the topology of $R$.

Let $\mathscr{D}=C(X) . \mathscr{D}$ is a maximal abelian subalgebra, actually a Cartan subalgebra (cf. [R], II.4.13), of $\mathscr{A}$. A norm-closed subalgebra $\mathscr{T}$ of $\mathscr{A}$ is called triangular if $\mathscr{T} \cap \mathscr{T}^{*}=\mathscr{D}$. A triangular subalgebra $\mathscr{T}$ of $\mathscr{A}$ is said to be strongly maximal if $\left(\mathscr{T}+\mathscr{T}^{*}\right)^{-}=\mathscr{A}$.

It follows from [MS], Theorem 4.1 that any strongly maximal triangular subalgebra of $\mathscr{A}$ is supported on a clopen subset $P$ of $R$, in the sense that $\mathscr{T}=C(P)$ where

$$
C(P)=\{f \in \mathscr{A}: f \text { vanishes on } R \backslash P\} .
$$

In the case of a strongly maximal triangular subalgebra $\mathscr{T}$ of an $\mathrm{AF} \mathrm{C}^{*}$-algebra, $P=\bigcup\{\hat{v}: v$ is a matrix unit in $\mathscr{T}\}$, that is, $(x, y) \in P$ iff there is some matrix unit $v \in \mathscr{T}$ such that $\sigma_{v}(x)=y . P$ has the following properties:

(1) $P \cap P^{-1}=X$

(2) $P \cdot P \subset P$

(3) $P \cup P^{-1}=R$

where ${ }^{-1}$ and $\cdot$ are the groupoid operations for $R$. Thus $P$ is the graph of a "total" order on $X$ (total only on the orbits of $R$ ).

A strongly maximal triangular subalgebra $\mathscr{T}=C(P)$ of $\mathscr{A}$ is called analytic if there is $d \in Z^{1}(R, \mathbb{R})$ such that $P=d^{-1}([0, \infty))$, that is $\mathscr{T}$ is supported on the set where the cocycle is non-negative. We usually say in this case that $\mathscr{T}$ corresponds to $d$ or that $\mathscr{T}$ is induced by $d$.

Let us view the UHF algebra $\mathscr{A}$ as the inductive limit of a sequence of matrix algebras (of size $N(n)$, if we want them to correspond to the factorization of $X$ given at the beginning of the section), and assume that the strongly maximal triangular subalgebra $\mathscr{T}$ of $\mathscr{A}$ has the form $\operatorname{Lim}_{\rightarrow}\left(\mathscr{T}_{n}, \varphi_{n}\right)$, where $\mathscr{T}_{n}$ are the $N(n) \times N(n)$ upper triangular matrices. In this context, whenever we choose a system of matrix units $\left\{e_{i j}^{(n)}\right\}$, we will always assume that

$$
\mathscr{T}_{n}=\operatorname{span}\left\{e_{i j}^{(n)}: 1 \leq i \leq j \leq N(n)\right\},
$$

$$
\mathscr{T}=\left(\operatorname{span}\left\{e_{i j}^{(n)}: \quad 1 \leq i \leq j \leq N(n), \quad n=1,2, \ldots\right\}\right)^{-}
$$


and we will say that the system corresponds, or is associated, to $\mathscr{T}$.

The reader is referred to [MS], [P2], [PPW2], [R] and [V] for more information about groupoid $\mathrm{C}^{*}$-algebras and their triangular subalgebras.

\section{COCYCLES THAT ARE NOT OF PRODUCT TYPE}

In this section we give two groups of examples of cocycles that are nonproduct type. The motivation for both examples came from triangular UHF algebra theory, but the proofs we give in this section are independent of that theory. For simplicity, we only consider here the case of the $2^{\infty}$ UHF algebra for the first set of examples. However, the examples and the proofs that follow can be easily generalized to arbitrary UHF algebras.

Perhaps the simplest coboundary on $R$ that we can write down is the one implemented by the "trace" function on $X, b(x)=\sum_{n=1}^{\infty} x_{n} / 2^{n}$. The cocycle of Example 3.1 is basically that one, except that we reverse the order in the cylindrical sets corresponding to the $n$-tuples of the form $(1,0, \ldots, 0)$.

Example 3.1. We construct the cocycle $d_{1}$ as follows. Let $Y_{i}=\{0,1\}$ and for $\left(i_{1}, \ldots, i_{n}\right) \in \prod_{i=1}^{n} Y_{i}$, let $P\left(i_{1}, \ldots, i_{n}\right)$ denote the $n$-cylindrical set

$$
\left\{x \in X: x_{1}=i_{1}, \ldots, x_{n}=i_{n}\right\}
$$

and let $\chi_{n}$ denote the characteristic function of the $n$-cylindrical set $P\left(i_{1}, \ldots, i_{n}\right)$, where $i_{1}=1$ and $i_{m}=0$ for $1<m \leq n$.

Consider the function on $X$ :

$$
b_{1}(x)=\frac{x_{1}}{2}+\sum_{n=1}^{\infty}\left[\left(1-\chi_{n}(x)\right) \frac{x_{n+1}}{2^{n+1}}+\chi_{n}(x)\left(\frac{1-x_{n+1}}{2^{n+1}}\right)\right] .
$$

Clearly $b_{1}$ is a continuous function on $X$. We define the cocycle

$$
d_{1}(x, y)=b_{1}(y)-b_{1}(x) \text {. }
$$

We are going to prove that $d_{1}$ is not of product type.

Theorem 3.2. The cocycle $d_{1}$ defined in.(3.1.2) is not of product type.

We show that $d_{1}$ is not locally constant. We will need the following string of lemmas. The first shows that $d_{1}$ vanishes only on the diagonal of $R$.

Lemma 3.3. $d_{1}(x, y)=0$ iff $x=y$, that is $b$ is one-to-one on the orbits in $R$. Proof. It is clear that $x=y$ implies that $d_{1}(x, y)=0$. We show the converse.

First note that $d_{1}(x, y)=0$ iff

$$
\left\{\begin{array}{l}
x_{1}=y_{1} \quad \text { and } \\
\left(1-\chi_{n}(y)\right) y_{n+1}+\chi_{n}(y)\left(1-y_{n+1}\right) \\
=\left(1-\chi_{n}(x)\right) x_{n+1}-\chi_{n}(x)\left(1-x_{n+1}\right) \text { for } n=1,2, \ldots .
\end{array}\right.
$$

Hence, we only need to show that (3.3.1) implies that $x=y$. We do that by induction.

Note first that (3.3.1) implies that $x_{1}=y_{1}$. Assume that $x_{i}=y_{i}$, for $i=1, \ldots, m$. Then $\chi_{m}(x)=\chi_{m}(y)$. If both are zero, then (3.3.1) yields $x_{m+1}=y_{m+1}$. If both are one, (3.3.1) becomes $1-y_{m+1}=1-x_{m+1}$. Hence, $x_{m+1}=y_{m+1}$ in any case. 
Lemma 3.4. $b_{1}(x)=1$ iff $x=(1,0, \ldots)$, that is $x_{1}=1$ and $x_{n}=0$ for $n>1$.

Proof. Clearly, $b_{1}(x)=1$ for $x=(1,0, \ldots)$. In order to show the converse, notice that $b_{1}(x)=1$ iff

$$
\left\{\begin{aligned}
x_{1} & =1 & & \text { and } \\
\left(1-\chi_{n}(x)\right) x_{n+1}+\chi_{n}(x)\left(1-x_{n+1}\right) & =1 & & \text { for } n=1,2, \ldots .
\end{aligned}\right.
$$

Just as in the previous lemma, it suffices to show that (3.4.1) implies that $x=$ $(1,0, \ldots)$.

An easy induction, along the lines of the one in the proof of Lemma 3.3, shows that (3.4.1) implies that $x_{1}=1$ and $\chi_{n}(x)=1$, for $n>1$. Hence $x_{1}=1$ and $x_{n}=0$ for $n=2, \ldots$.

Lemma 3.5. $b_{1}(x)=0$ iff $x=(0,0, \ldots)$, that is, $x_{n}=0$ for all $n$. Proof. Clearly $b_{1}(x)=0$ if $x=(0,0, \ldots)$, and also $b_{1}(x)=0$ iff (3.5.1) $\quad x_{1}=0$ and $\left(1-\chi_{n}(x)\right) x_{n+1}+\chi_{n}(x)\left(1-x_{n+1}\right)=0, \quad n=1,2, \ldots$

As before, a simple induction shows that (3.5.1) implies that $x_{n}=0$ for all $n$, so $b_{1}(x)=0$ implies $x=(0,0, \ldots)$.

We give now the proof of Theorem 3.2.

Proof. From Lemmas 3.4 and 3.5, it follows that $d_{1}$ takes the value 1 only at the point $((0,0, \ldots),(1,0, \ldots))$. Hence, $d_{1}$ is not locally constant, and therefore it cannot be of product type.

We move to our second set of examples. We will require that all the factors $Y_{i}$ of $X$, with the possible exception of the first, have the same cardinality $k$. This cocycle is constructed by applying the trace function not to $x$, but to the point whose $n$-th coordinate is the sum of the first $n$ coordinates of $x$, mod $k$. The motivation for this construction comes from triangular TUHF algebra theory and it will be explained in the next section.

Example 3.6. Let $Y_{1}=\{0,1, \ldots, N(1)-1\}$, and $Y_{n}=\{0,1, \ldots, k-1\}$, so that $\left|Y_{n}\right|=k$ for $n=2,3, \ldots$, where $k$ is some fixed positive integer. Consider

$$
b_{2}(x)=\frac{x_{1}}{N(1)}+\sum_{j=2}^{\infty} \frac{1}{N(j)}\left[\left(\sum_{i=1}^{j} x_{i}\right)(\bmod k)\right]
$$

where $z(\bmod k)$ is the remainder of the division of $z$ by $k$.

Notice that

$$
b_{2}(x) \leq \frac{N(1)-1}{N(1)}+\sum_{j=2}^{\infty} \frac{k-1}{N(j)}=1
$$

so the series defining $b_{2}$ converges uniformly on $X$, and hence $b_{2}$ is a continuous function.

We define the cocycle $d_{2}(x, y)=b_{2}(y)-b_{2}(x)$.

We will prove that $d_{2}$ is not of product type. 
Theorem 3.7. $d_{2}$ is not of product type.

As in Example 3.1, we prove the theorem by showing that $d_{2}$ is not locally constant. As before, the proof is reduced to a string of lemmas, the first of which shows that $d_{2}$ vanishes only on the diagonal $X$ of $R$.

Lemma 3.8. $d_{2}(x, y)=0$ iff $x=y$.

Proof. Clearly $x=y$ implies $d_{2}(x, y)=0$. It is also clear that $d_{2}(x, y)=0$ iff

$$
x_{1}=y_{1} \text { and }\left(\sum_{i=1}^{n} y_{i}\right)(\bmod k)=\left(\sum_{i=1}^{n} x_{i}\right)(\bmod k), \quad n=2, \ldots
$$

We show, by induction, that (3.8.1) implies that $x_{n}=y_{n}, n=1,2, \ldots$. Clearly (2.8.1) implies $x_{1}=y_{1}$. Assume $x_{i}=y_{i}$ for $i=1,2, \ldots, m$. From $\left(\sum_{i=1}^{m+1} y_{i}\right)(\bmod k)=\left(\sum_{i=1}^{m+1} x_{i}\right)(\bmod k)$, we get that $y_{m+1} \equiv x_{m+1}(\bmod k)$, and as $x_{m+1}, y_{m+1} \in\{0,1, \ldots, k-1\}$, we have that $x_{m+1}=y_{m+1}$.

Lemma 3.9. $b_{2}(x)=1$ iff

(1) $x_{1}=N(1)-1$

(2) $x_{1}+x_{2} \equiv k-1(\bmod k) ;$ and

(3) $x_{n}=0$ for $n=3,4, \ldots$.

Proof. If $x_{1}=N(1)-1, \quad x_{2}+x_{1} \equiv k-1(\bmod k)$ and $x_{n}=0$, for $n=$ $3,4, \ldots$, then an easy calculation shows that $b_{2}(x)=1$.

Now $b_{2}(x)=1$ iff

(3.9.1) $x_{1}=N(1)-1$ and $\left(\sum_{i=1}^{n} x_{i}\right) \equiv k-1(\operatorname{modk})$ for $n=2,3, \ldots$

An induction similar to the ones in Lemmas 3.3, 3.4 and 3.5, shows that (3.9.1) implies that $x_{1}=N(1), x_{1}+x_{2} \equiv k-1(\bmod k)$ and $x_{i}=0$ for $i=$ $3,4, \ldots$.

Lemma 3.10. $b_{2}(x)=0$ iff $x_{n}=0, n=1,2, \ldots$

Proof. If $x_{n}=0, n=1,2, \ldots$, it is clear that $b_{2}(x)=0$. Note that $b_{2}(x)=0$ iff

$$
x_{1}=0 \quad \text { and } \quad\left(\sum_{i=1}^{n} x_{i}\right) \equiv 0(\bmod k) \text { for } n=2,3, \ldots .
$$

By induction, we see that $(3.10 .1)$ implies that $x_{n}=0$ for $n=2,3, \ldots$ so the proof is complete.

We are ready to prove Theorem 3.7.

Proof of Theorem 3.7. From Lemmas 3.9 and 3.10, we see that $d_{2}$ takes the value 1 only at one point, so $d_{2}$ is not locally constant. Therefore $d_{2}$ is not of product type.

Remark 3.11. The one-parameter groups of automorphisms induced by $d_{1}$ and $d_{2}$, respectively, are nonproduct type. In order to see this, let us recall that given 
a cocycle $d$ on $R$, the one-parameter group of automorphisms $\alpha_{t}$ of $\mathscr{A}=$ $C^{*}(R)$ induced by $d$ is obtained by extending to $C^{*}(R)$ the automorphisms

$$
\alpha_{t}(f)(x, y)=\exp (\operatorname{itd}(x, y)) f(x, y) \quad \text { for } f \in C_{c}(R)
$$

where $C_{c}(R)$ is the algebra of continuous functions on $R$, with compact support ([R], Section II.5). If we express $\mathscr{A}=C^{*}(R)$ as $\bigcup \mathscr{A}_{n}$, where $\mathscr{A}_{n}$ are the finite-dimensional $\mathrm{C}^{*}$-algebras corresponding to the groupoids $X_{n} \times X_{n}$ (with $\left.X_{n}=\prod_{i=1}^{n} Y_{i}\right)$, then $C_{c}(R)=\bigcup_{i=1}^{\infty} \mathscr{A}_{n}$. We also recall that, for a UHF algebra, $d$ is a coboundary iff the induced automorphism group is inner ([R], Proposition II.5.3), and that a one-parameter automorphism group is induced by a cocycle iff it restricts to the identity on the diagonal $\mathscr{D}=C(X)$ ([V], Corollary 3.7). In particular, all automorphism groups induced by cocycles are representable in the sense of [HR].

A one-parameter group $\alpha$ of automorphisms of the UHF algebra $\mathscr{A}$ is of product type if it can be factored as a product of automorphisms of matrix algebras along a decomposition of $\mathscr{A}$ as an infinite tensor product of matrix algebras (see [B], Theorem 2.1, for the precise definition). Now, a factorization of $\mathscr{A}$ as an infinite product of matrix algebras corresponds to a factorization of $X$ as a product of finite sets. Thus, $\alpha$ is of product type iff $d$ is of product type. It follows from Theorems 3.2 and 3.7, that the automorphisms induced by $d_{1}$ and $d_{2}$ are both nonproduct type actions of $\mathbb{R}$. Both actions are inner.

\section{THE TUHF ALGEBRAS}

In this section we exhibit the TUHF algebras associated with the cocycles of Examples 3.1 and 3.6 of the previous section.

We recall that a subalgebra $\mathscr{S}$ of $\mathscr{A}$ is analytic if there is a real valued continuous cocycle $d$, such that $\mathscr{S}$ consists of those elements of $\mathscr{A}$ that are supported on $d^{-1}([0, \infty))$ (cf. [MS], [PPW2] and [V]). We denote that algebra by $C\left(d^{-1}[0, \infty)\right)$. If the cocycle vanishes only on the unit space $X$ of $R$, the algebra $C\left(d^{-1}[0, \infty)\right)$ is also triangular, actually, strongly maximal triangular.

Let $M_{2^{n}}$ be the $2^{n} \times 2^{n}$ matrices, and let $\lambda_{n}: M_{2^{n}} \rightarrow M_{2^{n+1}}$ be given by

$$
\lambda_{n}\left(e_{i j}^{(n)}\right)=\left\{\begin{array}{l}
e_{2 i-1,2 j-1}^{(n+1)}+e_{2 i, 2 j}^{(n+1)} \text { if } i, j<2^{n} \text { or } i=j=2^{n}, \\
e_{2 i, 2^{n+1}-1}^{(n+1)}+e_{2 i-1,2^{n+1}}^{(n+1)} \text { if } i<2^{n}, j=2^{n}, \\
e_{2^{n+1}, 2 j-1}^{(n+1)}+e_{2^{n+1}-1,2 j}^{(n+1)} \text { if } i=2^{n}, j<2^{n} .
\end{array}\right.
$$

$\lambda_{n}$ satisfies that $\lambda_{n}\left(\mathscr{T}_{n}\right) \subseteq \mathscr{T}_{n+1}$, where $\mathscr{T}_{n}$ is the upper triangular $2^{n} \times 2^{n}$ matrices.

Note that $\lambda_{n}$ is essentially the refinement embedding, but we flip the last two rows and columns. $\lambda_{n}$ was introduced by Peters, Poon and Wagner ([PPW1], Example 4.4), and has also been considered by S. Power ([P2], 6.4).

We will show that the embeddings (4.1) and the cocycle $d_{1}$ of Example 3.1 induce the same strongly maximal TUHF algebra.

Theorem 4.2. Let $\mathscr{T}$ be $\operatorname{Lim}_{\rightarrow}\left(\mathscr{T}_{n}, \lambda_{n}\right)$, where $\mathscr{T}_{n}$ and $\lambda_{n}$ are defined in (4.1), and let $d_{1}$ be the cocycle of Example 3.1. Then $C\left(d_{1}^{-1}[0, \infty)\right)$ is isometrically isomorphic to $\mathscr{T}$.

Proof. We will define a system of matrix units $\left\{f_{i j}^{(n)}\right\}$ in $C\left(d^{-1}[0, \infty)\right)$, such that $\left\{f_{i j}^{(n)}\right\}$ satisfies 
(4.2.1) the embedding (4.1); and

(4.2.2) that the linear span of the upper triangular matrix units $f_{i j}^{(n)}, i \leq j$, is dense in $C\left(d_{1}^{-1}[0, \infty)\right)$.

The correspondence $f_{i j}^{(n)} \leftrightarrow e_{i j}^{(n)}$, the latter satisfying (4.1), extends to an isometric isomorphism between $\mathscr{T}$ and $C\left(d_{1}^{-1}[0, \infty)\right)$, completing the proof of the theorem.

Besides the notation of Example 3.1 and the proof of Lemma 2.2 let us also consider

$$
b_{1}^{(n)}(x)=\frac{x_{1}}{2}+\sum_{j=1}^{n-1}\left[\left(1-\chi_{j}(x)\right) \frac{x_{j+1}}{2^{j+1}}+\chi_{j}(x) \frac{\left(1-x_{j+1}\right)}{2^{j+1}}\right] .
$$

Let $\vec{\imath}=\left(i_{1}, \ldots, i_{n}\right)$ and let $b_{1}^{(n)}(\vec{\imath})$ denote the common value of $b_{1}^{(n)}$ on the cylindrical set $P(\vec{\imath})$.

Let $d_{1}^{(n)}=\delta b_{1}^{(n)}$, that is $d_{1}^{(n)}(x, y)=b_{1}^{(n)}(y)-b_{1}^{(n)}(x)$. Note that $d_{1}^{(n)}$ is constant on $P(\vec{\imath}) \times P(\vec{\jmath}) \cap R$ (where $\vec{\jmath}=\left(j_{1}, \ldots, j_{n}\right)$ ), with value $b_{1}^{(n)}(\vec{\jmath})-$ $b_{1}^{(n)}(\vec{\imath})$, on that set. Also, $d_{1}^{(n)} \rightarrow d_{1}$ uniformly on compact sets.

Consider $\vec{\imath}, \vec{\jmath} \in \prod_{i=1}^{n} Y_{i}$, and $\sigma(\vec{\imath} ; \vec{\jmath})$ as defined in (2.2.1). For

$$
i=2^{n} b_{1}^{(n)}(\vec{\imath})+1 \quad \text { and } \quad j=2^{n} b_{1}^{(n)}(\vec{\jmath})+1
$$

we define $f_{i j}^{(n)}$ to be the characteristic function of the graph $\sigma(\widehat{\vec{\imath} ; \vec{\jmath})}$ of $\sigma(\vec{\imath} ; \vec{\jmath})$. Notice that:

$$
\sigma(\widehat{\vec{\imath} ; \vec{\jmath}})=\sigma(\vec{\imath}, \widehat{0 ;} \vec{\jmath}, 0) \cup \sigma(\vec{\imath}, \widehat{1 ;} \vec{\jmath}, 1) .
$$

We begin by showing that all upper triangular $f_{i j}^{(n)}$,s are in $C\left(d^{-1}[0, \infty)\right)$. Lemma 4.3. $d_{1}(x, y)>0$ for $(x, y) \in \hat{f}_{i j}^{(n)}$ with $i<j$.

Proof. We show first, by induction, that given $f_{i j}^{(n)}, d_{1}^{(n+m)}(x, y)>0$ for all $(x, y) \in \hat{f}_{i j}^{(n)}$ and $m=0,1,2, \ldots$.

Let $\vec{\imath}$ and $\vec{j}$ be the $n$-tuples associated to $i$ and $j$ according to (4.2.4). If $m=0$, we have that $\hat{f}_{i j}^{(n)} \subseteq P(\vec{l}) \times P(\vec{\jmath}) \cap R$. As we have observed $d_{1}^{(n)}$ has constant value $b_{1}^{(n)}(\vec{\jmath})-b_{1}^{(n)}(\vec{\imath})$ on $P(\vec{\imath}) \times P(\vec{\jmath}) \cap R$. Since $i<j$, we get, from (4.2.4), that $b_{1}^{(n)}(\vec{l})<b_{1}^{(n)}(\vec{\jmath})$, and therefore that $d_{1}^{(n)}$ is positive on $f_{i j}^{(n)}$.

Assume $d_{1}^{(n+p)}(x, y)>0$ for $(x, y) \in \hat{f}_{i j}^{(n)}$ and $i<j$. Then $d_{1}^{(n+p)}(x, y)$ $\geq 1 / 2^{n+p}$ and $\left|d_{1}^{(n+p+1)}(x, y)-d_{1}^{(n+p)}(x, y)\right| \leq 1 / 2^{n+p+1}$. Therefore, $d_{1}^{(n+p+1)}(x, y)>0$ for any $(x, y) \in \hat{f}_{i j}^{(n)}$.

It follows that $d_{1}^{(n+m)}(x, y)>0$ for $(x, y) \in \hat{f}_{i j}^{(n)}$ with $i<j$, and $m=$ $0,1, \ldots$. Since $d_{1}^{(n+m)}$ converges uniformly on compact sets to $d_{1}$, we get that $d_{1}$ is nonnegative on $\hat{f}_{i j}^{(n)}$. But, as $\hat{f}_{i j}^{(n)} \cap X=\varnothing$, we conclude that $d_{1}$ is positive on $\hat{f}_{i j}^{(n)}$.

Next, we show that the upper triangular $f_{i j}^{(n)}$ have a dense span in $C\left(d_{1}^{-1}[0, \infty)\right)$. 
Proposition 4.4. $C\left(d_{1}^{-1}[0, \infty)\right)$ coincides with the closed linear span of

$$
\left\{f_{i j}^{(n)}: 1 \leq i \leq j \leq 2^{n}, n=1,2, \ldots\right\} .
$$

Proof. Lemma 4.3 gives us one of the inclusions. Hence, the triangular algebra $C\left(d_{1}^{-1}[0, \infty)\right)$ contains the closed linear span of the upper triangular $f_{i j}^{(n)}$ 's, which is a strongly maximal triangular algebra. Therefore, we must have the other inclusion as well.

In order to finish the proof of Theorem 4.2, we only need to show that the system $\left\{f_{i j}^{(n)}\right\}$ satisfies $(4.1)$

Let $i, j, \vec{\imath}, \vec{\jmath}$ as in (4.2.4). Then, by (4.2.5) we must have that

$$
f_{i j}^{(n)}=f_{k_{1} l_{1}}^{(n+1)}+f_{k_{2} l_{2}}^{(n+1)} \quad \text { where }\left\{\begin{array}{l}
k_{1}=2^{n+1} b_{1}^{(n+1)}(\vec{l}, 0)+1, \\
l_{1}=2^{n+1} b_{1}^{(n+1)}(\vec{\jmath}, 0)+1, \\
k_{2}=2^{n+1} b_{1}^{(n+1)}(\vec{l}, 1)+1, \\
l_{2}=2^{n+1} b_{1}^{(n+1)}(\vec{\jmath}, 1)+1 .
\end{array}\right.
$$

We show that this coincides with (4.1), by considering the following cases.

Case 1. If $i, j<2^{n}$, then (4.2.4) and (4.2.3) give that $\vec{\imath} \neq(1,0, \ldots$, $0) \neq \vec{\jmath}$, so $\chi_{n}(\vec{\imath})=\chi_{n}(\vec{\jmath})=0$. Hence, from (4.2.3), we obtain that for $p=0,1$

$$
\begin{aligned}
& b_{1}^{(n+1)}(\vec{\imath}, p)=b_{1}^{(n)}(\vec{\imath})+\frac{p}{2^{n+1}}, \\
& b_{1}^{(n+1)}(\vec{\jmath}, p)=b_{1}^{(n)}(\vec{\jmath})+\frac{p}{2^{n+1}} .
\end{aligned}
$$

Thus, for $p=0,1$, we must have

$$
\begin{aligned}
& 2^{n+1} b_{1}^{(n+1)}(\vec{\imath}, p)=2\left(2^{n} b_{1}^{(n)}(\vec{\imath})\right)+p=2 i-2+p, \\
& 2^{n+1} b_{1}^{(n+1)}(\vec{\jmath}, p)=2\left(2^{n} b_{1}^{(n)}(\vec{\imath})\right)+p=2 j-2+p .
\end{aligned}
$$

Therefore $f_{i j}^{(n)}=f_{2 i-1,2 j-1}^{(n+1)}+f_{2 i, 2 j}^{(n+1)}$ as desired.

Case 2. If $i=j=2^{n}$ then (4.2.4) and (4.2.3) give that $\vec{\imath}=\vec{\jmath}=(1,0, \ldots, 0)$, so $\chi_{n}(\vec{l})=\chi_{n}(\vec{\jmath})=1$ and from (4.2.3) we obtain

$$
\begin{aligned}
& b_{1}^{(n+1)}(\vec{\imath}, p)=b_{1}^{(n)}(\vec{\imath})+\frac{1-p}{2^{n+1}}, \\
& b_{1}^{(n+1)}(\vec{\jmath}, p)=b_{1}^{(n)}(\vec{\jmath})+\frac{1-p}{2^{n+1}} .
\end{aligned}
$$

Therefore $f_{i j}^{(n)}=f_{2^{n+1}, 2^{n+1}}^{(n+1)}+f_{2^{n+1}-1,2^{n+1}-1}^{(n+1)}$.

Case 3. If $i=2^{n}$ and $j<2^{n}$, then (4.2.4) and (4.2.3) give that $\vec{\imath}=(1,0, \ldots$, $0)$, and $\vec{\jmath} \neq(1,0, \ldots, 0)$. Thus $\chi_{n}(\vec{l})=1$ and $\chi_{n}(\vec{\jmath})=0$, so, for $p=0,1$ (4.2.3) yields

$$
\begin{aligned}
& b_{1}^{(n+1)}(\vec{\imath}, p)=b_{1}^{(n)}(\vec{\imath})+\frac{1-p}{2^{n+1}}, \\
& b_{1}^{(n+1)}(\vec{\jmath}, p)=b_{1}^{(n)}(\vec{\jmath})+\frac{p}{2^{n+1}} .
\end{aligned}
$$

Thus $f_{2^{n}, j}^{(n)}=f_{2^{n+1}, 2 j-1}^{(n+1)}+f_{2^{n+1}-1,2 j}^{(n+1)}$. 
Case 4. If $i<2^{n}$ and $j=2^{n}$, we show that (4.1) holds with an argument similar to the one of Case 3 .

Next, we exhibit the analytic TUHF algebra associated with Example 3.6.

Let $N(1)$ and $k$ be as in Example 3.6. Let $M_{n}$ be the $N(n) \times N(n)$ matrices, and let $\psi_{n}: M_{n} \rightarrow M_{n+1}$ be given by

$$
\psi_{n}\left(e_{i j}^{(n)}\right)=\sum_{q=1}^{k} e_{k(i-1)+q, k(j-1)+(q+j-i)(\bmod k)}^{(n+1)} .
$$

Note that if $\mathscr{S}_{n}$ is the algebra of $N(n) \times N(n)$ upper triangular matrices, then $\psi_{n}\left(\mathscr{S}_{n}\right) \subseteq \mathscr{S}_{n+1}$.

The embeddings $\psi_{n}$ are examples of nest embeddings, and they have been studied in [HP]. Here all the permutations involved in the embedding of the first superdiagonal are equal to the cyclic permutation $(12 \cdots k) \in S_{k}$, the permutations on $\mathrm{k}$ elements, so the family $\psi_{n}$ defines a homogeneous nest embedding ([HP], Definition 1.2).

We are going to show that the cocycle $d_{2}$ of Example 3.6 and the embeddings given by (4.6) induce the same strongly maximal TUHF algebra.

Theorem 4.7. Let $\mathscr{S}=\operatorname{Lim}_{\rightarrow}\left(\mathscr{S}_{n}, \psi_{n}\right)$, where $\mathscr{S}_{n}$ and $\psi_{n}$ are given by (4.6), and let $d_{2}$ be the cocycle of Example 3.6. Then $C\left(d_{2}^{-1}[0, \infty)\right)$ is isometrically isomorphic to $\mathscr{S}$.

Proof. As in the proof of Theorem 4.2, we will construct a system of matrix units in $C\left(d_{2}^{-1}[0, \infty)\right)$ such that it satisfies properties corresponding to (4.2.1) (for the embeddings (4.6)) and (4.2.2) (for the cocycle $d_{2}$ ). The same argument given in Theorem 4.2 will yield an isomorphism in the present case.

In addition to the notation of the proof of Lemma 2.1 and Example 3.6, consider

$$
b_{2}^{(n)}(x)=\frac{x_{1}}{N(1)}+\sum_{j=2}^{n} \frac{1}{N(j)}\left[\left(\sum_{i=1}^{j} x_{i}\right)(\bmod k)\right] .
$$

Let $\vec{\imath}=\left(i_{1}, \ldots, i_{n}\right)$ and $\vec{\jmath}=\left(j_{1}, \ldots, j_{n}\right)$. We denote by $b_{2}^{(n)}(\vec{\imath})$ the constant value of $b_{2}^{(n)}$ on $P(\vec{\imath})$. If $d_{2}^{(n)}=\delta b_{1}^{(n)}$, then, as in Theorem 4.2, $d_{2}^{(n)}$ is constant on the set $P(\vec{\imath}) \times P(\vec{\jmath}) \cap R$, with value $b_{2}^{(n)}(\vec{\jmath})-b_{2}^{(n)}(\vec{\imath})$, and $d_{2}^{(n)} \rightarrow d_{2}$ uniformly on compact sets.

For $\vec{\imath}, \vec{\jmath} \in \prod_{i=1}^{n} Y_{i}$, and $\sigma(\vec{\imath} ; \vec{\jmath})$ define

$$
i=N(n) b_{1}^{(n)}(\vec{\imath})+1 \quad j=N(n) b_{2}^{(n)}(\vec{\jmath})+1
$$

and set $f_{i j}^{(n)}$ to be the characteristic function of $\sigma(\widehat{\vec{l}, \vec{\jmath}})$. Notice that instead of (4.2.5) we have

$$
\sigma(\widehat{\vec{l}, \vec{\jmath}})=\bigcup_{p=0}^{k-1} \sigma(\vec{\imath}, \widehat{p ;} \vec{\jmath}, p) .
$$

Now, an argument similar to the one we gave in Lemma 4.3, with $N(n)$ instead of $2^{n}$, establishes the following lemma. 
Lemma 4.8. $d_{2}(x, y)>0$ for $(x, y) \in \hat{f}_{i j}^{(n)}$ with $i<j$.

The same proof given for Proposition 4.4 will establish the following proposition.

Proposition 4.9. $C\left(d_{2}^{-1}[0, \infty)\right)$ coincides with the closed linear span of

$$
\left\{f_{i j}^{(n)}: 1 \leq i \leq j \leq N(n), \quad n=1,2, \ldots\right\} .
$$

We finish the proof of Theorem 4.7, by showing that $\left\{f_{i j}^{(n)}\right\}$ satisfies (4.6).

Let $i, j, \vec{i}, \vec{j}$ as in (4.7.2). Then, at the level of matrix units, (4.7.3) becomes:

$$
f_{i j}^{(n)}=\sum_{p=0}^{k-1} f_{N(n+1) b_{2}^{(n+1)}(\vec{l}, p)+1, N(n+1) b_{2}^{(n+1)}(\vec{\jmath}, p)+1}^{(n+1)}
$$

with

$$
\begin{aligned}
N(n+1) b_{2}^{(n+1)}(\vec{\imath}, p)+1 & =N(n+1)\left\{b_{2}^{(n)}(\vec{\imath})+\frac{\left[\left(p+\sum_{t=1}^{n} i_{t}\right)(\bmod k)\right]}{N(n+1)}\right\}+1 \\
& =k N(n) b_{2}^{(n)}(\vec{\imath})+\left(p+\sum_{t=1}^{n} i_{t}\right)(\bmod k)+1 \\
& =k(i-1)+\left(p+\sum_{t=1}^{n} i_{t}\right)(\bmod k)+1 \quad \text { by }(4.7 .2) .
\end{aligned}
$$

Similarly,

$$
N(n+1) b_{2}^{(n+1)}(\vec{\jmath}, p)+1=k(j-1)+\left(p+\sum_{t=1}^{n} j_{t}\right)(\bmod k)+1 .
$$

Note that $\left\{\left(p+\sum_{t=1}^{n} i_{t}\right)(\bmod k): p=0, \ldots, k-1\right\}=\{0,1, \ldots, k-1\}$ as the first set contains $k$ consecutive positive integers.

Thus, we only need to show that if $p \in\{0,1, \ldots, k-1\}$, then

$$
\left(p+\sum_{t=1}^{n} j_{t}\right) \equiv\left(j-i+p+\sum_{t=1}^{n} i_{t}\right)(\bmod k) .
$$

In order to do that, we need the following lemma.

Lemma 4.12. For any $x \in X$

$$
\left(N(n) b_{2}^{(n)}(x)+x_{n+1}\right) \equiv\left(\sum_{i=1}^{n} x_{i}+x_{n+1}\right)(\bmod k) .
$$

Proof. If $n=1$, then $N(1) b_{2}^{(n)}(x)+x_{2}=x_{1}+x_{2}$, so above equality holds. If $n>1$, from the definition of $b_{2}^{(n)}$ we see that

$$
\begin{aligned}
N(n) b_{2}^{(n)}(x)+x_{n+1} & =N(n)\left\{\frac{x_{1}}{N(1)}+\sum_{j=2}^{n} \frac{1}{N(j)}\left[\left(\sum_{i=1}^{j} x_{i}\right)(\bmod k)\right]\right\}+x_{n+1} \\
& =k^{n-1} x_{1}+\left\{\sum_{j=2}^{n} k^{n-j}\left[\left(\sum_{i=1}^{j} x_{i}\right)(\bmod k)\right]\right\}+x_{n+1} .
\end{aligned}
$$


Since $k^{n-1} x_{1} \equiv 0(\bmod k)$ and $k^{n-j}\left[\left(\sum_{i=1}^{j} x_{i}\right)(\bmod k)\right] \equiv 0(\bmod k)$, for $j<n$, it follows that

$$
N(n) b_{2}^{(n)}(x)+x_{n+1} \equiv\left(\sum_{i=1}^{n} x_{i}\right)(\bmod k)+x_{n+1} \equiv \sum_{i=1}^{n} x_{i}+x_{n+1} \quad(\bmod k)
$$

which proves the lemma.

Applying Lemma 4.12 to $x$ such that $x_{t}=i_{t}, t=1, \ldots, n$, and $x_{n+1}=$ $p, p=0, \ldots, k-1$, we get

$$
p+\sum_{t=1}^{n} i_{t} \equiv N(n) b_{2}^{(n)}(\vec{l})+p=(i-1)+p
$$

and similarly

$$
p+\sum_{t=1}^{n} j_{t} \equiv N(n) b_{2}^{(n)}(\vec{\jmath})+p=(j-1)+p
$$

Therefore

$$
p+\sum_{t=1}^{n} j_{t} \equiv p+\sum_{t=1}^{n} i_{t}+(j-i) \quad(\bmod k) .
$$

Thus (4.11) holds. Therefore, $\left\{f_{i j}^{(n)}\right\}$ satisfies (4.6).

\section{NONPRODUCT TYPE ANALYTICITY}

In the preceding sections we have exhibited examples of nonproduct type cocycles and we have constructed their coresponding analytic TUHF algebras. In this section we are going to show that the analytic TUHF algebras of Section 4 have the property that they do not correspond to any product type cocycle, that is their support cannot be expressed as $d^{-1}([0, \infty))$, where $d$ is a product type cocycle.

Some analytic TUHF algebras are canonically associated to product type cocycles, which makes them more tractable. Among them is the standard embedding TUHF algebra ([V], Example 6.1, [PPW2], Theorem 2.2). At the onset of this research, we thought that algebras like the standard embedding TUHF, corresponded only to product type cocycles. However, that is not the case, as B. Solel has obtained a nonproduct type cocycle that also corresponds to the standard embedding TUHF algebra.

We introduce the following definition.

Definition 5.1. Let $\mathscr{T}$ be a triangular analytic subalgebra of the UHF algebra $\mathscr{A}$. If there is a cocycle $d$ of product type such that $\mathscr{T}=C\left(d^{-1}[0, \infty)\right)$, we say that $\mathscr{T}$ is Product Type Analytic. We say that $\mathscr{T}$ is Nonproduct Type Analytic if it is not Product Type Analytic.

With the terminology just introduced, B. Solel has shown that not all the cocycles corresponding to a Product Type Analytic TUHF are of product type. We will show that there exist Nonproduct Type Analytic TUHF algebras. In fact, Theorem 5.3 implies that the analytic TUHF algebras of Examples 3.1 and 3.6 are Nonproduct Type Analytic. 
We also remark that in [V], Section 5, we used the term Product Type Analytic, without making it into an official definition, for those TUHF algebras that are associated (usually in a canonical way) with a product type cocycle. However, at the time, the results mentioned at the beginning of this section were not known, so it was not clear what the right definition should have been.

Let $d$ be a cocycle of product type on $R$, and let $\left\{e_{i j}^{(n)}: 1 \leq i, j \leq N(n), n=\right.$ $1,2, \ldots\}$ be a system of matrix units induced by $d$. Let $d(i, j ; n)$ denote the constant value of $d$ on $\hat{e}_{i j}^{(n)}$ (cf. Lemma 2.2 and Definition 2.3).

We will denote by $\sigma_{i j}^{(n)}$ the partial homeomorphism whose graph is $\hat{e}_{i j}^{(n)}$. The triangular algebra $C\left(d^{-1}[0, \infty)\right)$ induces an order in its diagonal matrices $\left\{e_{i i}^{(n)}\right\}$, called the diagonal order (cf. [PPW1], Section 3) or the algebraic order (cf. [P1], Section 1). We denote that order by $\preccurlyeq$. We consider that order restricted to the sets $X_{n}=\left\{e_{j j}^{(n)}: 1 \leq j \leq N(n)\right\}$, and will also regard $\sigma_{i j}^{(n)}$ as a permutation on the set $X_{n+k}$, when convenient. We can always assume that $e_{i i}^{(n)} \preccurlyeq e_{j j}^{(n)}$ iff $i \leq j$. That is, $d(i, j ; n) \geq 0$ iff $i \leq j$.

We recall the following result.

Lemma 5.2 (Lemma 5.5 and Lemma 5.6 of [V]). Let $d$ be a product type cocycle, $e_{i j}^{(n)}$ and $\sigma_{i j}^{(n)}$, as defined above. Consider $\sigma_{i j}^{(n)}$ with $i \leq j$. Then $\sigma_{i j}^{(n)}$ preserves the diagonal order induced by $C\left(d^{-1}[0, \infty)\right)$ on $X_{n+k}$ for $k \geq 0$. That is, if $\hat{e}_{p p}^{(m)}, \hat{e}_{q q}^{(m)} \subseteq \operatorname{range}\left(\hat{\sigma}_{i j}^{(n)}\right)$ (which is $e_{i i}^{(n)}$ ) for $m \geq n$, and $e_{p p}^{(m)} \preccurlyeq e_{q q}^{(m)}$, then $\sigma_{i j}^{(n)}\left(e_{p p}^{(m)}\right) \preccurlyeq \sigma_{i j}^{(m)}\left(e_{q q}^{(m)}\right)$.

S. Power has introduced the notions of strong normalizer and regular embeddings ([P1], Definitions 2.1 and 2.3 , respectively). With this terminology, Lemma 5.2 is saying that the matrix units induced by a product type cocycle are contained in the strong normalizer semigroup, and that the embeddings relating these matrices, are regular embeddings.

Lemma 5.2 has also an AF version, in which the hypothesis $d$ is of product type is substituted by $d$ is locally constant, and the conclusion is that for any point in the support of the triangular AF algebra, there is a matrix unit $v$ such $\hat{v}$ contains the point and $d$ is constant on $\hat{v}$ (see Remark 5.13).

We will use the following terminology in the next theorem. We say that $e_{p q}^{(m)}$ is a restriction of $e_{i j}^{(n)}$, if $\hat{e}_{p q}^{(m)} \subseteq \hat{e}_{i j}^{(n)}$, that is, $\sigma_{p q}^{(m)}$ is a restriction of $\sigma_{i j}^{(n)}$. We also say that a sequence of matrix units $\left\{e_{k}: k=1,2, \ldots\right\}$ is nested, if $\hat{e}_{1} \supseteq \hat{e}_{2} \supseteq \ldots$.

We come to the main result of this section.

Theorem 5.3. Let $\mathscr{T}=\operatorname{Lim}_{\rightarrow}\left(\mathscr{T}_{n}, \varphi_{n}\right)$ be a TUHF algebra, where $\mathscr{T}_{n}$ is the $N(n) \times N(n)$ upper triangular matrices. Let $\left\{e_{i j}^{(n)}: 1 \leq i, j \leq N(n), n=\right.$ $1,2, \ldots\}$ be a system of matrix units associated with $\mathscr{T}$. Assume that there is a nested sequence of matrix units $\left\{e_{k}^{\left(n_{k}\right)}\right\}$ among the $\left\{e_{i j}^{(n)}\right\}$ such that $\sigma_{k}^{\left(n_{k}\right)}$ does not preserve the order of $X_{n_{k}+1}$. Then $\mathscr{T}$ cannot have a system of matrix units such that their corresponding partial homeomorphisms preserve the diagonal order induced by $\mathscr{T}$. In particular, if $\mathscr{T}$ is analytic, then $\mathscr{T}$ is Nonproduct Type Analytic.

Proof. $\left\{\hat{e}_{k}^{\left(n_{k}\right)}\right\}$ is a nested sequence of compact sets, so it has a nonempty inter- 
section. Moreover, as any system of matrix units forms a base for the topology of $R$, the intersection of the sequence $\left\{\hat{e}_{k}^{\left(n_{k}\right)}\right\}$, has only one point. Let $(a, b)$ be that point.

By Lemma 5.2, $\left\{e_{i j}^{(n)}\right\}$ cannot be the system of matrix units induced by a product type cocycle. Let $\left\{f_{i j}^{(m)}\right\}$ be another system of matrix units such that $\mathscr{T}=\left(\operatorname{span}\left\{f_{i j}^{(m)}: i \leq j, m=1,2, \ldots\right\}\right)^{-}$. We will show that there is no cocycle having constant values on all the $f_{i j}^{(m)}$, by showing that there is a matrix unit, among the $f_{i j}^{(m)}$, such that it does not preserve the diagonal order on the set of minimal projections contained in its range.

Let $f_{m t}^{(l)}$ be such that $(a, b) \in f_{m t}^{(l)}$. Find $k$ such that $\hat{e}_{k}^{\left(n_{k}\right)} \subseteq \hat{f}_{m t}^{(l)}$. As $\sigma_{k}^{\left(n_{k}\right)}$ does not preserve the order of $X_{n_{k}+1}$, there are minimal projections $e_{p p}^{\left(n_{k}+1\right)}$, $e_{r r}^{\left(n_{k}+1\right)}, e_{s s}^{\left(n_{k}+1\right)}$ and $e_{q q}^{\left(n_{k}+1\right)}$ such that

$$
e_{p p}^{\left(n_{k}+1\right)} \preccurlyeq e_{r r}^{\left(n_{k}+1\right)} \preccurlyeq e_{s s}^{\left(n_{k}+1\right)} \preccurlyeq e_{q q}^{\left(n_{k}+1\right)}
$$

while $\hat{e}_{p q}^{\left(n_{k}+1\right)}, \hat{e}_{r s}^{\left(n_{k}+1\right)} \subseteq \hat{e}_{k}^{\left(n_{k}\right)} \subseteq \hat{f}_{m t}^{(l)}$.

Pick $x \in \hat{e}_{p p}^{\left(n_{k}+1\right)}, y \in \hat{e}_{r r}^{\left(n_{k}+1\right)}, w \in \hat{e}_{s s}^{\left(n_{k}+1\right)}$ and $z \in \hat{e}_{q q}^{(n+1)}$ such that

$$
x<y, w<z \text { and }(x, z) \in \hat{e}_{p q}^{\left(n_{k}+1\right)}, \quad(y, w) \in \hat{e}_{r s}^{\left(n_{k}+1\right)} .
$$

Let $u, v$ be matrix units in the system $\left\{f_{i j}^{(m)}\right\}$, such that $(x, y) \in \hat{u} \subseteq \hat{e}_{p q}^{\left(n_{k}+1\right)}$, and $(w, y) \in \hat{v} \subseteq \hat{e}_{r s}^{\left(n_{k}+1\right)}$. Thus $\hat{u}, \hat{v} \subseteq \hat{f}_{m t}^{(l)}$. By considering the initial and final projections of $u$ and $v$, we see that $f_{m t}^{(l)}$ does not preserve the diagonal order on the set of minimal projections contained in the final projection $f_{m m}^{(l)}$ of $f_{m t}^{(l)}$. In fact (5.3.1) and (5.3.2) imply that $u u^{*} \preccurlyeq v v^{*} \preccurlyeq v^{*} v \preccurlyeq u^{*} u$, with the partial homeomorphism induced by $f_{m t}^{(l)}$ mapping $u u^{*} \mapsto u^{*} u$ and $v v^{*} \mapsto v^{*} v$. Thus no cocycle can have constant values on each of the $\hat{f}_{i j}^{(m)}$, and therefore the system $\left\{f_{i j}^{(m)}\right\}$ is not induced by a product type cocycle.

It is interesting to notice that the proof of Theorem 5.3 holds also for strongly maximal triangular AF algebras. However, for AF algebras, the proof shows that the strongly maximal triangular algebra $\mathscr{T}$ cannot correspond to a locally constant cocycle. In Remark 5.13 we expand on the AF version of Theorem 5.3 .

In the remainder of this section, we obtain some consequences of Theorem 5.3, among them, that the TUHF's of Sections 3 and 4 are Nonproduct Type Analytic.

Corollary 5.4. Let $\mathscr{T}$ be an analytic TUHF algebra. If there is only one $x \in X$ such that the orbit $[x]$ of $x$ has a largest element and a smallest element in the order induced by $\mathscr{T}$ on $X$, then $\mathscr{T}$ is Nonproduct Type Analytic.

Proof. Let $\left\{e_{i j}^{(n)}: 1 \leq i, j \leq N(n), n \geq 1\right\}$ be a system of matrix units associated to $\mathscr{T}$. Let $y$ (resp. $z$ ) be the smallest (resp. largest) element in $[x]$. Then $y \in e_{11}^{(n)}$ and $z \in e_{N(n), N(n)}^{(n)}$ for all $n$.

Choose $n_{0}$ such that $\sigma_{1, N\left(n_{0}\right)}^{\left(n_{0}\right)}(y)=z$. Then, some restriction of $\sigma_{1, N\left(n_{0}\right)}^{\left(n_{0}\right)}$ among the $\sigma_{i j}^{\left(n_{0}+1\right)}$ should map $y$ to $z$. That can only be $\sigma_{1, N\left(n_{0}+1\right)}^{\left(n_{0}+1\right)}$. Moreover, 
$\sigma_{1, N\left(n_{0}\right)}^{\left(n_{0}\right)}$ does not preserve the order of $X_{n_{0}+1}$. In fact, if $e_{p q}^{\left(n_{0}+1\right)}$ is another restriction of $e_{1, N\left(n_{0}\right)}^{\left(n_{0}\right)}$, we have that $e_{N\left(n_{0}+1\right), N\left(n_{0}+1\right)}^{\left(n_{0}+1\right)} \succcurlyeq e_{q q}^{\left(n_{0}+1\right)} \succcurlyeq e_{p p}^{\left(n_{0}+1\right)} \succcurlyeq e_{11}^{\left(n_{0}+1\right)}$. Proceeding inductively, we conclude that $\left\{e_{1, N\left(n_{0}+k\right)}^{\left(n_{0}+k\right)}: k=0,1, \ldots\right\}$ is a nested sequence of matrix units such that their corresponding partial homeomorphisms do not preserve the diagonal order. By Theorem 5.3, $\mathscr{T}$ is Nonproduct Type Analytic.

Corollary 5.5. $\mathscr{T}$, the TUHF algebra defined by the embeddings (4.1), is Nonproduct Type Analytic.

Proof. Theorem 4.2 shows that $\mathscr{T}$ is analytic. $(0,0, \ldots)$ and $(1,0,0, \ldots)$ are the smallest and largest elements, respectively, of their common orbit, for the order induced by the cocycle $d_{1}$ (cf. Lemmas 3.4 and 3.5). Moreover, that orbit is the only one with a smallest and a largest elements. Thus, by Corollary $5.4, \mathscr{T}$ is Nonproduct Type Analytic.

We should remark that in [V], Example 6.4, we showed, using different techniques, that $\mathscr{T}$ cannot be associated to a product type cocycle. At that time, we did not know that $\mathscr{T}$ is analytic.

In order to show that the TUHF algebra given by the embeddings (4.6) is Nonproduct Type Analytic, we need the following terminology.

Definition 5.6. An embedding $\nu: M_{n} \rightarrow M_{n \cdot k}$ (here $M_{m}$ are the $m \times m$ complex matrices), given by $\nu\left(a_{i j}\right)=\left(a_{i j} u_{i j}\right)$, where each $u_{i j}$ is a permutation $k \times k$ matrix, is called a Nest embedding. If $u_{i j}=\pi^{j-i}$, where $\pi$ is a fixed permutation $k \times k$ matrix, we say that $\nu$ is a homogeneous nest embedding. A subalgebra $\mathscr{T}$ of a UHF algebra $\mathscr{A}$ is called a Full nest subalgebra if $\mathscr{T}=$ $\operatorname{Lim}_{\rightarrow}\left(\mathscr{T}_{n}, \varphi_{n}\right)$, where $\mathscr{T}_{n}$ is the $N(n) \times N(n)$ upper triangular matrices, and $\varphi_{n}$ is a nest embedding.

Notice that the homogeneous nest embedding corresponding to the permutation $\pi$ on $\{1,2, \ldots, k\}$ is given by

$$
e_{i j}^{(n)}=\sum_{p=1}^{k} e_{k(i-1)+p, k(j-1)+\pi^{j-i}(p)}^{(n+1)} .
$$

We will use the notation

$$
\varphi_{n}\left(e_{i j}^{(n)}\right)=u_{i j}^{(n)} \otimes e_{i j}^{(n)}
$$

to denote the nest embedding $\varphi_{n}$ in terms of the matrix units $e_{i j}^{(n)}$ of a system associated with the full nest algebra $\operatorname{Lim}_{\rightarrow}\left(\mathscr{T}_{n}, \varphi_{n}\right)$.

Remark 5.7. The terminology of Definition 5.6 is due to Hopenwasser and Peters ([HP], Definitions 1.1-1.3).

As an example of nest embeddings, we have the ones defined by (3.1). In this case, the number $k$ of Definition 5.6 is 2 , for all the embeddings, and the matrices $u_{i j}^{(n)}$ are:

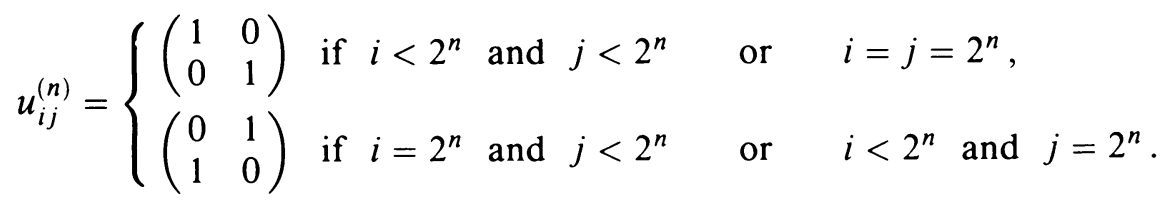


The following definition departs from the terminology of Hopenwasser and Peters.

Definition 5.8. Let $\mathscr{T}=\operatorname{Lim}_{\rightarrow}\left(\mathscr{T}_{n}, \varphi_{n}\right)$ satisfy

(1) $\mathscr{T}_{n}$ is the upper triangular $N(n) \times N(n)$ matrices;

(2) there is a positive integer $k$ satisfying $N(n+1)=k N(n)$ for all $n$; and

(3) for all $n, \varphi_{n}$ is the homogeneous nest embedding corresponding to a permutation $\pi$ of $\{1,2, \ldots, k\}$.

$\mathscr{T}$ is called a stationary homogeneous nest embedding TUHF algebra.

As an example of a stationary homogeneous nest embedding TUHF algebra, we have the TUHF algebra $\mathscr{S}$ defined by the embeddings (4.6), which corresponds to the $k$-cycle $\pi=(12 \ldots k)$ on $\{1, \ldots, k\}$. Note that $\pi^{j-i}(p)=$ $((j-i)+p)(\bmod k)$, so $(5.6 .1)$ for this particular $\pi$ coincides with the definition (4.6) of the embeddings for $\mathscr{S}$.

The TUHF algebra $\mathscr{T}$, defined by (4.1), however, is not a stationary homogeneous nest embedding TUHF.

In order to show that $\mathscr{S}$ is Nonproduct Type Analytic, we show that all stationary homogeneous nest embedding TUHF algebras corresponding to the $k$-cycles $\left(\begin{array}{lll}1 & 2 \ldots k\end{array}\right)$, are Nonproduct Type Analytic.

Lemma 5.9. Let $\mathscr{T}=\operatorname{Lim}_{\rightarrow}\left(\mathscr{T}_{n}, \varphi_{n}\right)$ be a nest embedding TUHF algebra. If $u_{i j}^{(n)} \neq I_{k}$ (the identity matrix), then $\sigma_{i j}^{(n)}$ does not preserve the order of $X_{n+1}$.

Proof. Let $k=N(n+1) / N(n)$, and assume that $u_{i j}^{(n)} \neq I_{k}$. Note that $\sigma_{i j}^{(n)}$, viewed as a map from $\left\{e_{(i-1) k+p}^{(n+1)}: p=1, \ldots, k\right\}$ (whose sum is $e_{i i}^{(n)}$ ) to $\left\{e_{(j-1) k+p}^{(n+1)}: p=1, \ldots, k\right\}$ (whose sum is $\left.e_{j j}^{(n)}\right)$, satisfies

$$
\sigma_{i j}^{(n)}\left(e_{(i-1) k+p}^{(n+1)}\right)=e_{(j-1) k+q}^{(n+1)} \quad \text { if } \quad u_{i j}^{(n)}\left(\mathbf{v}_{q}\right)=\mathbf{v}_{p}
$$

where $\left\{\mathbf{v}_{1}, \ldots, \mathbf{v}_{k}\right\}$ is the standard orthonormal basis of $\mathbb{R}^{k}$.

Let $Y=\left\{p: \sigma_{i j}^{(n)}\left(e_{(i-1) k+p}^{(n+1)}\right) \neq e_{(j-1) k+p}^{(n+1)}\right\} . \quad Y$ is nonempty, as $u_{i j} \neq I_{k}$. Let $p_{0}$ be the smallest element in $Y$. Then

$$
\sigma_{i j}^{(n)}\left(e_{(i-1) k+p_{0}}^{(n+1)}\right)=e_{(j-1) k+q}^{(n+1)} \text { for some } q>p_{0}
$$

Let $p_{1}$ be such that $\sigma_{i j}^{(n)}\left(e_{(i-1) k+p_{1}}^{(n+1)}\right)=e_{(j-1) k+p_{0}}^{(n+1)}$. Then $p_{0}<p_{1}$, and we have that

$$
e_{(i-1) k+p_{0}}^{(n+1)} \preccurlyeq e_{(i-1) k+p_{1}}^{(n+1)} \quad \text { but } \quad \sigma_{i j}^{(n)}\left(e_{(i-1) k+p_{0}}^{(n+1)}\right) \succcurlyeq \sigma_{i j}^{(n)}\left(e_{(i-1) k+p_{1}}^{(n+1)}\right)
$$

since $\sigma_{i j}^{(n)}\left(e_{(i-1) k+p_{0}}^{(n+1)}\right)=e_{(j-1) k+q}^{(n+1)}$ and $\sigma_{i j}^{(n)}\left(e_{(j-1) k+p_{1}}^{(n+1)}\right)=e_{(j-1) k+p_{0}}^{(n+1)}$.

Theorem 5.10. Let $\mathscr{T}=\operatorname{Lim}_{\rightarrow}\left(\mathscr{T}_{n}, \varphi_{n}\right)$ be a stationary homogeneous embedding TUHF algebra, where $\varphi_{n}$ is associated to the $k$-cycle $\left(\begin{array}{llll}1 & 2 & \ldots & k\end{array}\right)$. Then $\mathscr{T}$ is Nonproduct Type Analytic.

Proof. Let $k$ be the positive integer such that $N(n+1)=k N(n)$, and let $\pi$ be the $k$-cycle. Define

$$
i_{1}=1, \quad j_{1}=2 \text { and } i_{n+1}=k\left(i_{n}-1\right)+1, \quad j_{n+1}=k\left(j_{n}-1\right)+2 .
$$


We show by induction that $\pi^{j_{n}-i_{n}}=\pi \neq I_{k}$, the identity $k \times k$ matrix. In fact, $\pi^{j_{1}-i_{1}}=\pi \neq I_{k}$, and

$$
\pi^{j_{m+1}-i_{m+1}}=\pi^{k\left(j_{m}-i_{m}\right)+1}=\pi \neq I_{k} .
$$

It follows from (5.6.1), that $\left\{e_{i_{n} j_{n}}^{(n)}: n=1,2, \ldots\right\}$ is a nested sequence. Since $\pi^{j_{n}-i_{n}}=\pi \neq I_{k}$, we also have that $\sigma_{i_{n} j_{n}}^{(n)}$ does not preserve the diagonal order in $X_{n+1}$. Thus, Theorem 4.3 gives that $\mathscr{T}$ is Nonproduct Type Analytic.

Remark 5.11. Notice that it follows from ([PPW1], Theorem 4.6), that all Full nest algebras are trivially analytic. In particular, this proves that the TUHF algebras of Theorems 4.2 and 4.7 are analytic. However, the cocycle obtained in [PPW1], Theorem 3.16, although applicable to any trivially analytic TAF algebra, is not easy to construct in practice, mainly because it involves an enumeration of all the increasing clopen subsets. The cocycles of Example 3.1 and 3.6 are not examples of the generic cocycles given in [PPW1], Theorem 3.16. This generic cocycle was not helpful for the results of this paper for two reasons. First, the already mentioned complication of dealing with an enumeration of the clopen increasing sets, makes it difficult to express the generic cocycle in terms of the coordinates of the points of $X$, and therefore eliminates any possibility of obtaining direct proofs (like the ones in Section 3) that the cocycles are not of product type. Second, the generic cocycle of [PPW1], Theorem 3.16 is not linked to a representation of its corresponding analytic algebra (like Examples 3.1 and 3.6 are linked to the embeddings (4.1) and (4.6) respectively), in a way that will allow the study of this algebras that we have carried out in this section.

Combining our Theorem 5.3 and Lemma 5.9 with [PPW1], Theorem 4.6, we obtain the following theorem regarding Full nest algebras.

Theorem 5.12. Let $\mathscr{T}=\operatorname{Lim}_{\rightarrow}\left(\mathscr{T}_{n}, \varphi_{n}\right)$ be a Full nest algebra, with $\varphi_{n}\left(e_{i j}^{(n)}\right)=$ $u_{i j}^{(n)} \otimes e_{i j}^{(n)}$ for the matrix units in a system associated with $\mathscr{T}(c f .(5.6 .2))$. If there is a nested sequence of matrix units $e_{i_{s} j_{s}}^{\left(n_{s}\right)}$ such that $u_{i_{s} j_{s}}^{\left(n_{s}\right)} \neq I$ (the identity matrix of the size of $u_{i_{s} j_{s}}^{\left(n_{s}\right)}$ ), then $\mathscr{T}$ is Nonproduct Type Analytic.

Proof. By [PPW1], Theorem 4.6, $\mathscr{T}$ is analytic, and by Theorem 5.3 and Lemma 5.9 it is Nonproduct Type Analytic.

Remark 5.13. As indicated after their proofs, Lemma 5.2 and Theorem 5.3 have AF algebra versions. Product type notions are exclusive to UHF algebras, and there is no generalization of that concept to AF algebras. However, after this paper was submitted, we became aware of recent work by A. Donsig and T. Hudson [DH], which indicates that, for triangular AF algebra theory, locally constant cocycles will constitute an appropriate generalization to AF algebras of the notion of product type cocycles for UHF algebras. We wish to indicate what the AF versions of the above mentioned results are, first, because the proofs are virtually the same, and, second, because the hypothesis of Theorem 5.3, namely that there is a sequence of normalizers that do not preserve the order and such that the corresponding sequence of supports shrinks down to a point, also appear, in a slightly different formulation, in the recent work of Donsig and Hudson [DH]. This seems to indicate that said hypothesis will probably play an important rôle in triangular AF algebra theory. 
First, for Lemma 5.2, notice that the proof depends only on the fact that a product type cocycle is locally constant. So, for AF algebras, the same proof will show a version of Lemma 5.2 in which (a) the hypothesis $d$ is product type will be substituted by the $d$ is locally constant; (b) the matrix unit system can be any matrix unit system for the enveloping AF algebra; and (c) the conclusion will be that for each point there is some matrix unit that contains the point and that preserves the order.

For Theorem 5.3, the same proof establishes that if $\mathscr{T}$ is a strongly maximal triangular AF algebra with a nested sequence of normalizers that do not preserve the order, such that the sequence of supports shrinks down to a point, then $\mathscr{T}$ cannot correspond to a locally constant cocycle.

Note that if a strongly maximal triangular AF algebra $\mathscr{T}$ satisfies the hypothesis of Theorem 5.3, which can also be formulated as the support of $\mathscr{T}$ has a point $(x, y)$ with a neighborhood base consisting of supports of non-orderpreserving normalizers, then $(x, y)$ cannot belong to the support of a normalizer that preserves the order. Thus $\mathscr{T}$ is not generated by its order preserving normalizers. The converse is also true, so the hypothesis of Theorem 5.3 is equivalent to

$\mathscr{T}$ is not generated by its order preserving normalizers.

Thus an equivalent formulation of the AF version of Theorem 5.3 reads as follows: if $\mathscr{T}$ satisfies (5.14), then $\mathscr{T}$ cannot correspond to a locally constant cocycle. The embeddings (4.1) and (4.6) show non-locally-constant analytic TAF algebras.

Donsig and Hudson [DH] have shown that the negative of (5.14) implies that $\mathscr{T}$ is determined, up to isometric isomorphism, by its lattice of ideals.

\section{REFERENCES}

[B] O. Bratteli, Crossed products of UHF algebras by product type actions, Duke Math. J. 46 (1979), 1-23.

[DH] A. Donsig and T. Hudson, The ideal lattice of triangular AF algebras (work in progress).

[HR] D. Handelman and W. Rossmann, Actions of compact groups on AF C*-algebras, Illinois J. Math. 29 (1985), 51-95.

[HP] A. Hopenwasser and J. Peters, Full nest algebras, Illinois J. Math. 38 (1994).

[K] A. Kishimoto, On the fixed point algebra of a UHF algebra under a periodic automorphism of product type, Publ. Res. Inst. Math. Sci. Kyoto 13 (1977), 777-791.

[MS] P. Muhly and B. Solel, Subalgebras of groupoid $C^{*}$-algebras, J. Reine Angew. Math. 402 (1989), 41-75.

[PPW1] J. Peters, Y. Poon and B. Wagner, Triangular AF algebras, J. Operator Theory 23 (1990), $81-114$.

[PPW2] - Analytic TAF algebras, Canad J. Math. 45 (1993), 1009-1031; Erratum, Canad. J. Math. 46 (1994), 395-396.

[P1] S. Power, Algebraic orders on $K_{0}$ and approximtely finite operator algebras, J. Operator Theory 27 (1992), 87-106.

[P2] - Limit algebras: an introduction to subalgebras of $C^{*}$-algebras, Pitman Research Notes in Math. Ser., No. 278, Longman Scientific \& Technical, UK, 1992. 
[R] J. Renault, $A$ groupoid approach to $C^{*}$-algebras, Lecture Notes in Math., vol. 793, SpringerVerlag, Berlin, 1980.

[S] B. Solel, Unpublished notes.

[V] B. Ventura, Strongly maximal triangular AF algebras, Internat. J. Math. 2 (1991), 567-598.

Department of Mathematics. California State University. San Bernardino, CaliforNIA 92407

E-mail address: bventura@illey.csusb.edu 